\title{
La couleur en prothèse faciale
} (3e partie)
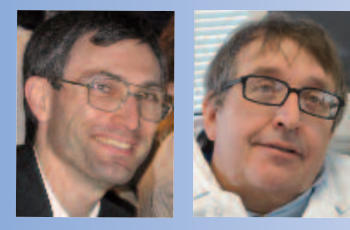

Christophe BONNEFOY

Diplômé universitaire

de Prothèse faciale appliquée,

78 , avenue Aristide-Briand,

92160 Antony.

\section{Jacques DICHAMP}

Praticien hospitalier,

Coordinateur de 1995 à 2010

du Diplôme universitaire

de Prothèse faciale appliquée,

Faculté de médecine Pierre-et-Marie-Curie,

Paris 6,

Service de stomatologie

et de chirurgie maxillo-faciale,

47-83, boulevard de l'Hôpital,

75651 Paris cedex 13.
L'importance de cette publication, tant par son sujet que par son volume, a incité le Comité de Rédaction à la scinder en trois parties.
La première partie à été publiée dans le $n^{\circ} 254$ de mars 2011, la deuxième dans le $n^{\circ} 255$ de juin 2011.
AOS 2012;257:5-15

DOI: $10.1051 /$ aos/2012102

(C) EDP Sciences
- pigments
- biocompatibilité
- technique du portrait
- palette du prothésiste facial
- maquette
- maquillage des prothèses au fauteuil 


\section{Les problèmes spécifiques rencontrés en prothèse faciale}

\section{Problèmes liés \\ à la solidité des pigments}

La solidité des pigments à la lumière se doit d'être maximale en prothèse faciale et conditionne sans contredit la palette que nous proposerons. Ainsi les pigments les plus purs et les plus finement broyés sont les seuls à prendre à en considération conformément à la dénomination de " couleurs à l'huile extrafines ". Les pigments doivent être choisis essentiellement d'après leur résistance aux UV (sur une échelle de 1 à 3 étoiles) et il est souhaitable, lorsque le fabricant le mentionne, de choisir en outre ceux qui passeront l'épreuve de la stabilité chimique dans les mélanges avec succès. Par exemple, jamais de blanc d'argent (céruse contenant du plomb) avec des pigments au cadmium susceptibles de noircissement ultérieurs assez fâcheux... Chez Lefranc \& Bourgeois, les couleurs compatibles entre elles sont repérées par un « $M$ » rouge.

\section{Problème de la qualité du subjectile}

La prothèse faciale est un subjectile bien particulier qui justifie une spécialité picturale à part entière. Ce n'est pas de la peinture hyperréaliste mais cosmétique à visée mimétique. Le matériau est bien différent de ceux que les peintres emploient habituellement et pourrait déplaire à plus d'un titre quant à ses médiocres qualités. La prothèse se comporte donc comme un mauvais subjectile par rapport au bois ou à la toile. La pérennité est donc contestable surtout si l'on tient compte de l'effet des agents atmosphériques.

Il faut atteindre le degré de translucidité des couches épithélio-conjonctives (fig. 28).

Une prothèse, surtout si elle est en silicone, a tendance par transparence à laisser deviner les cavités chirurgicales sous-jacentes. Elle ne peut être considérée comme un support opaque ordinaire (bois ou toile). C'est même la raison d'être de ce cours spécialisé. Le fond absorbe certaines radiations lumineuses et en renvoie d'autres, d'où la gêne esthétique.

On conseille par conséquent d'arrêter les rayons lumineux grâce à un fond opaque blanc ou ocre de chair claire teint dans la masse afin de constituer un fond opaque et clair qui sera présent à l'endroit de l'intrados prothétique. Le blanc de titane est le blanc dont l'indication est excellente puisqu'il est le plus couvrant et le plus opaque des blancs connus.

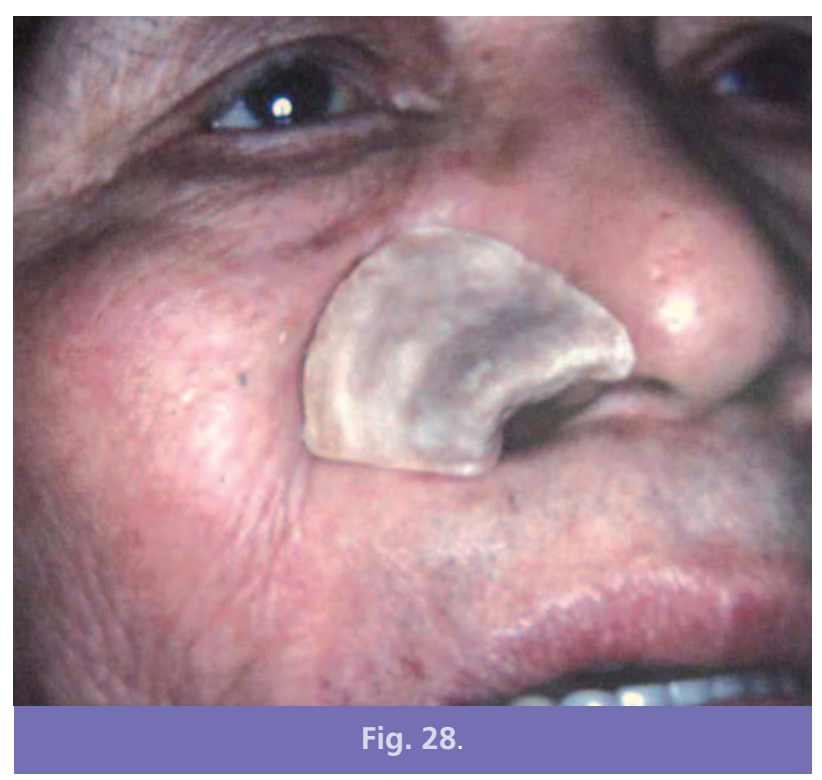


Notons par ailleurs que le silicone manifeste une nette tendance au jaunissement avec le temps, ce qui compromet la bonne tenue des couleurs : le silicone ne satisfait pas aux critères essentiels de choix d'un bon subjectile dans l'état actuel des connaissances même si les recherches sur les silicones améliorent constamment leur stabilité dans le temps. Ils conservent relativement bien leurs propriétés élastiques et thixotropiques, résistent mieux aux chocs thermiques et à la plupart des solvants agressifs. Enfin, ils sont beaucoup plus résistants vis-à-vis des liquides organiques et à leurs attaques enzymatiques que les résines qui ne s'emploient plus désormais que dans les prothèses transitoires.

C'est pourquoi les industriels ont travaillé sur ces points précis pour offrir aux professionnels des kits de coloration intrinsèques par incorporation de pigments dans le matériau avant la coulée dans le moule du silicone, par exemple avec le Drop Ortho ${ }^{\circledR}$, silicone réticulant par addition qui durcit en une vingtaine de minutes. Il fournit alors une ébauche qui se doit d'être la plus aboutie possible.

Cette façon de procéder nécessite une grande maîtrise pour ne pas avoir à retoucher en technique de coloration extrinsèque, cette dernière appliquant toute la puissance du rendu hyperréaliste étudié ci-dessus. Avec un œil exercé, ce procédé de retouche, familier des peintres, produit indiscutablement les meilleurs résultats.

Toutefois, sur le plan théorique, comme en peinture à I'huile traditionnelle, moins on applique de couches successives, plus la longévité de la prothèse sera augmentée.

La technique de coloration extrinsèque ne devrait donc être qu'une "roue de secours " si ce n'était son exceptionnelle efficacité clinique...
Cette technique utilise une forme particulière de silicone RTV (Room Temperature Vulcanizing) polymérisant en couches minces : la colle à froid (CAF). Le Drop Ortho $^{\circledR}$ se liquéfie sous I'effet d'une huile de silicone ayant une action solvante sur les pigments. Certains produits comme le FE-100 jouent ce rôle de diluant synthétique qui possède l'avantage d'être réputé inoffensif pour les pigments extrinsèques. $A$ contrario, le trichloréthane, qui a remplacé le cyclohexane tant décrié pour sa toxicité, ouvre efficacement les pores du silicone et permet à la peinture à l'huile de pénétrer en surface, réalisant ainsi un véritable maquillage. Après évaporation, laquelle permet de respecter automatiquement la règle $\mathrm{du}$ " souple sur rigide ", le matériau silicone est censé retrouver toutes ses qualités.

Enfin, ils sont capables de réaliser une empreinte quasi parfaite à quelques $\mu$ près de la peau du patient et en particulier de ses pores, avec un résultat extrêmement mimétique.

Voici donc quelques informations qui appellent une réflexion : la technique de coloration en prothèse faciale n'a en vérité rien qui la différencie réellement de la technique de la peinture à I'huile traditionnelle des grands maîtres à partir du moment où l'on soigne l'exécution pour réaliser une œuvre optimisée dans la pérennité. Un artiste ne désire pas voir son œuvre craqueler dans les mois qui suivent sa création : il en est de même pour le prothésiste facial.

Il faut donc respecter les protocoles et en particulier les temps de séchage entre les couches tout en diminuant au maximum leur nombre. L'homologie de structure entre les CAFs et le silicone sous-jacent est un élément incontestablement rassurant en faveur des kits de colorations extrinsèques de prothèse faciale. Toutefois la peinture à l'huile, malgré son côté 
artisanal, parfois regardée avec condescendance, est d'une efficacité qui a fait ses preuves. Il suffit d'aller dans les musées pour rapidement s'en persuader et constater notamment que la technique des primitifs italiens (et leur fameuse " transparence ») a su si bien résister à l'usure du temps. Les traces d'huile présentes dans les pigments ne peuvent pas altérer profondément le subjectile silicone. En revanche, les pigments eux-mêmes, indépendamment de leur innocuité ou de leur toxicité potentielles, ne peuvent être exclus d'une interaction avec le silicone lors de la prise (oxydes de manganèse en particulier mais aussi macromolécules aromatiques polycyclophénanthrènes). Ce problème touche toute approche esthétique de coloration des épithèses.

En définitive, ce sujet, très vaste, ne fait qu'alimenter une discussion particulièrement technique qui nécessiterait des expérimentations cliniques ultérieures, notamment comparatives, dont la littérature est assez avare.

\section{Problèmes liés à la technique du trompe-l'oeil}

Le trompe-l'œil accentue l'impression de relief et est presque constamment utilisé en prothèse faciale. C'est en quelque sorte une « accentuation 3D ». La boule est un excellent solide imaginaire qui permet l'étude de ce style pictural tout à fait intéressant en prothèse faciale en théorie. En pratique, il faut user de cette " théorie des ombres " avec une certaine prudence. On reste dans le mimétisme.

En prothèse, on choisit d'accentuer un éclairage à peu près frontal, c'est-à-dire lorsque la prothèse est frappée par un rayonnement ayant tendance à écraser au maximum les formes.

Le portrait en clair-obscur, caractéristique de la technique du trompe-l'œil chère à l'expressionnisme baroque du Caravage, a connu son heure de gloire au début du XVIIe siècle. Ici, nous montrons un détail du Jeune garçon mordu par un lézard de 1515 (fig. 29).

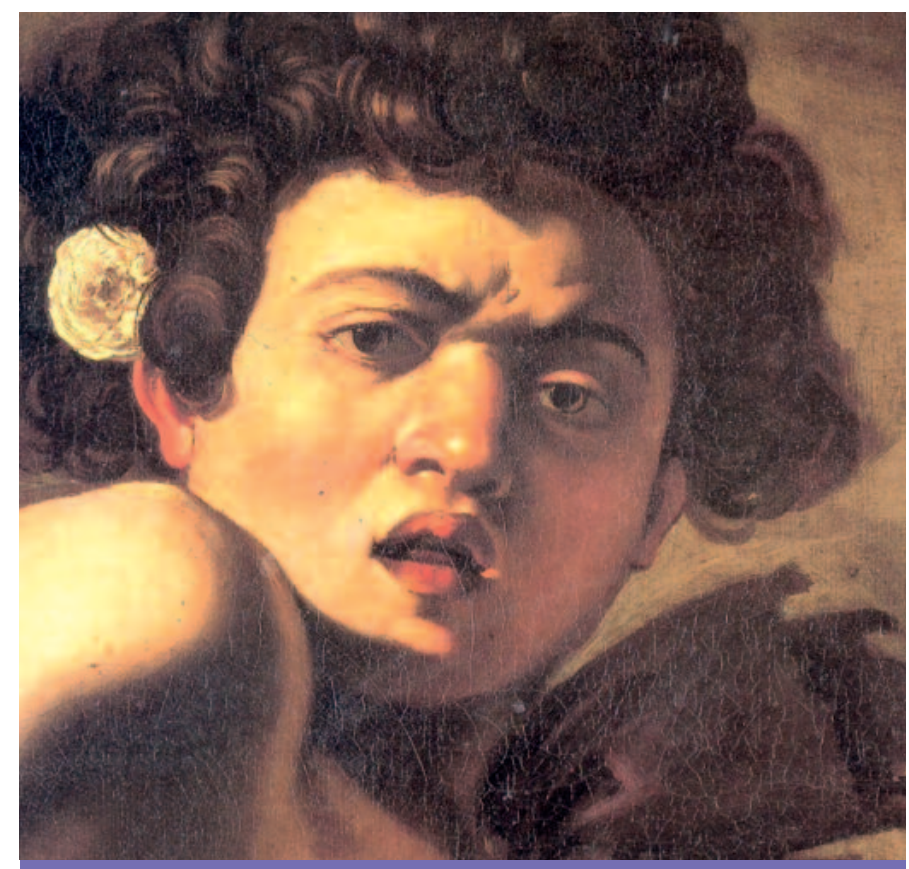

Fig. 29 Le Caravage, Jeune garçon mordu par un lézard. Détail. 


\section{Problèmes liés}

à la biocompatibilité des matériaux

Le silicone répond aux normes européennes de biocompatibilité des matériaux à usage externe. Les pigments sont ceux des industries spécialisées, généralement acrylo-vinyliques, et satisfont aux exigences des normes européennes. Ces normes touchent également les pigments pour peintures à l'huile et les produits toxiques s'y trouvent bannis progressivement.
La cancérogenèse, et en particulier la mutagenèse et la tératogenèse, semblent être écartées de facto par l'usage externe qui n'impose que très peu de contact avec les muqueuses et le milieu interstitiel, ce qui limite considérablement leur diffusion dans l'organisme.

Seuls problèmes : I'allergie au niveau des bords de l'épithèse qui est à traiter au cas par cas mais qui concerne plus souvent le silicone ou la résine acrylique du subjectile, voire les matériaux des colles destinées à améliorer la rétention, que celle induite par les pigments.

\section{La palette du portraitiste}

La palette standard des artistes-peintres comporte en général une dizaine de couleurs et celle des portraitistes souvent un peu moins.

Fort heureusement, le portrait est une spécialité " couleur chair » qui n'est pas ce qu'il y a de plus difficile à rendre en peinture à condition de respecter les règles édictées ci-dessus.

Afin de pouvoir dégager une palette comportant les pigments chimiquement les plus fixes à la lumière et les plus stables dans les mélanges, indiqués respectivement par trois étoiles et un « $M$ » rouge sur les tubes de peintures à I'huile extrafines Lefranc \& Bourgeois, il est nécessaire de connaître les spécificités chimiques des différents constituants.

\section{Rappels}

La classification des poudres colorantes comporte trois catégories de pigments :

- les couleurs naturelles généralement minérales;

- les couleurs artificielles d'origine minérale ou synthétique ;
- les laques qui sont des extraits tintoriaux d'origine animale, végétale, minérale et même synthétique.

Les nouvelles normes européennes exigent une stabilité des pigments et une moindre toxicité. La tendance des maisons fabriquant les pigments à I'huile est de remplacer les pigments jugés de solidité douteuse ou réputés coûteux à fabriquer par des mélanges de colorants organiques divers obtenus par synthèse chimique. Ils bénéficient ainsi des dernières avancées dans le domaine des peintures acrylo-vinyliques. Ainsi ces mélanges de pigments remplacent une couleur bien connue depuis longtemps dans la palette du peintre comme le bleu turquoise ou la laque de garance. Le cas du jaune de Naples est un cas d'école : traditionnellement constitué d'un mélange antimoniate de plomb et sulfate de chaux, sa composition s'est modifiée et le plomb a disparu en faveur de la benzymidazolone et du chrome (fig. 30).

Mais si les fabricants se doivent de porter l'appellation « imitation », ils ne le font pas toujours. Cependant les artistes chevronnés se rendent 
bien compte que ces nouvelles couleurs réagissent différemment dans les mélanges et en particulier avec le blanc. Elles sont d'une manière générale plus violentes et plus difficiles à doser dans les mélanges. Cela aura des conséquences directes très problématiques pour le prothésiste facial qui devra faire très attention aux pigments qu'il manie et veiller à n'utiliser ceux-ci qu'en petite quantité, c'est-àdire une " pointe » comme on a coutume de le dire dans le jargon des peintres.

\section{La palette du prothésiste facial}

La palette de l'école de prothèse faciale du service de stomatologie et chirurgie maxillo-faciale

\begin{tabular}{|c|c|c|c|c|}
\hline \multicolumn{5}{|c|}{ YELLOW/ORANGE } \\
\hline 207 & Cadmium yellow lemon & Cadmium zinc sulfide & PY35 & 77205 \\
\hline 208 & Cadmium yellow light & Cadmium zinc sulfide & PY35 & 77205 \\
\hline 210 & Cadmium yellow deep & & PY35 & $\begin{array}{l}77205 \\
77202\end{array}$ \\
\hline \multirow{2}{*}{211} & Cadmium orange & $\begin{array}{l}\text { Cadmium seleno-sulfide } \\
\text { Cadmium seleno-sulfide }\end{array}$ & PO20 & 77202 \\
\hline & & Cadmium zinc sulfide & PY35 & 77205 \\
\hline \multirow[t]{3}{*}{222} & Naples yellow light & Zinc oxide & PW4 & 77947 \\
\hline & & Benzimidazolone & PY 154 & 11781 \\
\hline & & Antimony/chromium/itanium oxide & $\mathrm{PBr} 24$ & 77310 \\
\hline \multirow[t]{3}{*}{223} & Naples yellow deep & Zinc oxide & PW4 & 77947 \\
\hline & & Benzimidazolone & PY154 & 11781 \\
\hline & & Antimony/chromium/titanium oxide & PBR24 & 77310 \\
\hline \multirow[t]{3}{*}{224} & Naples yellow red & Zinc oxide & PW4 & 77947 \\
\hline & & Antimony/chromium/itanium oxide & PBr24 & 77310 \\
\hline & & Diketopyrrolo-pyrrole & PO43 & 71105 \\
\hline 227 & Yellow ochre & Synthetic iron oxide & PY42 & 77492 \\
\hline 228 & Yellow ochre light & Antimony/chromium/itanium oxide & PBr24 & 77310 \\
\hline 231 & Gold ochre & Synthetic iron oxide & PY 42 & 77492 \\
\hline 232 & Orange ochre & Synthetic iron oxide & PY42 & 77492 \\
\hline \multirow[t]{2}{*}{234} & Raw sienna & Synthetic iron oxide & PY42 & 77492 \\
\hline & & Synthetic iron oxide & PR101 & 77491 \\
\hline 242 & Aureoline & Arylide & PY 150 & 12764 \\
\hline 251 & Stil de grain yellow & Isoindolinone & PY110 & 56280 \\
\hline 254 & Perm. lemon yellow & Bismuth vanadium & PY184 & $\ldots$ \\
\hline 265 & Transp oxide yellow & Synthetic iron oxide & PY42 & 77492 \\
\hline \multirow[t]{2}{*}{266} & Permanent orange & Diketopyrrolo-pyrrole & PO73 & $\ldots$ \\
\hline & & Benzi & PY154 & 11781 \\
\hline 271 & Cadmium yellow medium & Cadmium zinc sulfide & PY35 & 77205 \\
\hline 272 & Transp. yellow medium & Arylide & PY128 & 20037 \\
\hline \multirow[t]{2}{*}{273} & Transp. oxide orange & Synthetic iron oxide & PY 42 & 77492 \\
\hline & & Synthetic iron oxide & PR101 & 77491 \\
\hline \multirow[t]{2}{*}{279} & Nickel titan yeliow light & Zinc oxide & PW4 & 77947 \\
\hline & & Bismuth vanadium & PY184 & $\cdots$ \\
\hline \multirow[t]{3}{*}{280} & Nickel titan.yellow deep & Zinc oxide & PW4 & 77947 \\
\hline & & Benzimidazolone & PY154 & 11781 \\
\hline & & Antimony/chromium/titanium oxide & PBr24 & 77310 \\
\hline 281 & Transp.yellow green & Azomethine & PY129 & 48042 \\
\hline \multirow[t]{3}{*}{282} & Naples yellow green & Zinc oxide & PW4 & 77947 \\
\hline & & Synthetic iron oxide & PY42 & 77492 \\
\hline & & Anhydrous chromium sesquioxide & PG17 & 77288 \\
\hline & Permanent yellow light & Benzimidazolone & PY154 & 11781 \\
\hline \multirow[t]{2}{*}{284} & Perm yellow medium & Benzimidazolone & PY154 & 11781 \\
\hline & & Diketopyrrolo-pyrrole & $\mathrm{PO} 43$ & 71105 \\
\hline \multirow[t]{2}{*}{285} & Permanent yellow deep & Benzimidazolone & PY154 & 11781 \\
\hline & & Diketpyrrolo-pyrrole & $\mathrm{PO} 43$ & 71105 \\
\hline
\end{tabular}

Fig. 30 . 
de la Pitié-Salpêtrière est assez simple, elle fait appel à quelques pigments (fig. 31 ) :

- jaune de cadmium ;

- rouge de cadmium ;

- bleu outremer pour les ombres et les nævi en association au rouge de cadmium ;

- bleu de cobalt pour les ombres en transparence :

- terre de sienne naturelle qui sert à la teinte de base de la prothèse (obtenue par vitropression) sortie du moule avec une pointe de rouge de cadmium ou de terre de sienne brûlée ;

- terre de sienne brûlée ;

- blanc de titane à utiliser avec parcimonie car il opacifie les mélanges, tout le contraire de la transparence recherchée.

Notons que le diluant utilisé est de la résine auto qui sèche très vite et demande donc une certaine expérience et un certain doigté. On peut également utiliser le trichloréthylène ou le trichloréthane. Le cyclohexane est désormais interdit car jugé trop toxique.

Enfin, un artifice courant en prothèse faciale consiste à utiliser des flocs :

- marron, pour simuler le duvet sur la peau et matifier celle-ci lors de la couche de finition similaire à celle du vernis d'un tableau en l'associant à du silicone médical $\mathrm{A}$;

- rouges, pour simuler les veinules et toute la vascularisation bien utile pour simuler les varicosités chez les patients ayant subi des radiothérapies de la sphère oro-faciale ;

Pour que les couleurs prennent toute leur transparence, il faut travailler en glacis et en fondus : c'est l'inverse de ce que font les débutants et les peintres qui veulent attirer l'attention avec des

\begin{tabular}{|c|c|c|}
\hline COULEURS & PIGMENTS & DOMAINE D'UTILISATION \\
\hline BLEUS & $\begin{array}{l}\text { Bleu de Cobalt, } \\
\text { Bleu Outremer }\end{array}$ & Ombres, glacis pour griser, verres, veines, naevi \\
\hline OCRES & $\begin{array}{l}\text { Terre de Sienne naturelle, } \\
\text { Terre de Sienne brulée }\end{array}$ & $\begin{array}{l}\text { Teinte de base avec pointe de rouge et de jaune } \\
\text { et blanc de titane. } \\
\text { Terre de Sienne brulée pour peau bronzée }\end{array}$ \\
\hline ROUGES & $\begin{array}{l}\text { Rouge de cadmium } \\
\text { Terre de Sienne brulée }\end{array}$ & $\begin{array}{l}\text { Une pointe pour la vascularisation, varicosités et } \\
\text { rougissement d'une teinte de base trop jaune }\end{array}$ \\
\hline JAUNES & Jaune de cadmium & $\begin{array}{l}\text { Éclaircir et réchauffer les rouges en luttant } \\
\text { contre la tendance au rosissement, bronzage }\end{array}$ \\
\hline POURPRES & $\begin{array}{l}\text { Rouge de cadmium } \\
+ \text { bleu de cobalt }\end{array}$ & $\begin{array}{l}\text { Ombres violacées, vascularisation, naevi avec } \\
\text { de l'outremer }\end{array}$ \\
\hline VERTS & $\begin{array}{l}\text { Jaune de cadmium } \\
+ \text { bleu de cobalt }\end{array}$ & Ombres sur peaux bronzées, cuivrées. Barbes \\
\hline BLANCS & Titane & $\begin{array}{l}\text { "Le » Blanc de référence de par sa grande opacité } \\
\text { et son pouvoir couvrant fond opaque. } \\
\text { Mais attention au manque de transparence } \\
\text { qu'il engendre en trop grande quantité }\end{array}$ \\
\hline
\end{tabular}

Fig. 31. 
couleurs criardes. Ne jamais oublier que la prothèse faciale est faite pour se faire oublier, non pour se faire admirer dans un musée entre de nombreux concurrents.

En pratique, on procède de la sorte :

- placer le blanc au centre d'une assiette en carton blanche, les différentes couleurs étant organisées tout autour selon le cercle chromatique ;

- utiliser deux pinceaux, un pour les couleurs claires, un pour les couleurs foncées. Bien nettoyer ceux-ci à chaque changement de couleur. Ceci permet de respecter le contraste des valeurs en ne salissant pas le mélange avec des couleurs inattendues ;

- créer les couleurs complexes par ajout d'un pigment, voire deux, et d'une pointe de la couleur se rapprochant de la complémentaire de la couleur donnée par le ou les pigments choisis. Ceci permet de griser les teintes et de moduler finement la dose de couleur complémentaire mélangée en créant des fondus tout en délicatesse faits avec du « presque rien ». Attention, cet exercice n'est plus pratiqué dans les écoles d'Art et n'est pas destiné aux débutants car il faut « avoir l'œil » comme on dit, c'est-à-dire savoir inter- rompre le travail et se reculer pour juger de I'effet obtenu ;

- bien attendre le séchage avant de passer à la couche suivante.

Il faut savoir que peindre à l'intérieur d'une pièce métallique moulée par galvanoplastie est infiniment plus mimétique : I'effet de transparence est garanti à la manière de la peau sur les tissus sous-jacents mais le coût de la technique est élevé.

L'évaporation du diluant au fur et à mesure du travail permet de respecter automatiquement la règle " gras sur maigre ", les reprises ultérieures devant être limitées au maximum, ne serait-ce que pour ne pas déformer l'anatomie de la pièce prothétique.

La technique de maquillage des épithèses au fauteuil ne nécessite pas que de bons outils et de la patience, elle nécessite aussi un sens artistique poussé afin de personnaliser la prothèse par rapport aux tissus avoisinants ou du côté sain (fig. 32). Plus la perte de substance est importante, plus ces derniers repères se font rares.

Il est indispensable de reproduire les particularités de la peau, ou plutôt ses imperfections pour ne pas obtenir une impression in fine de poupée de son : taches vasculaires, naevi, pig-

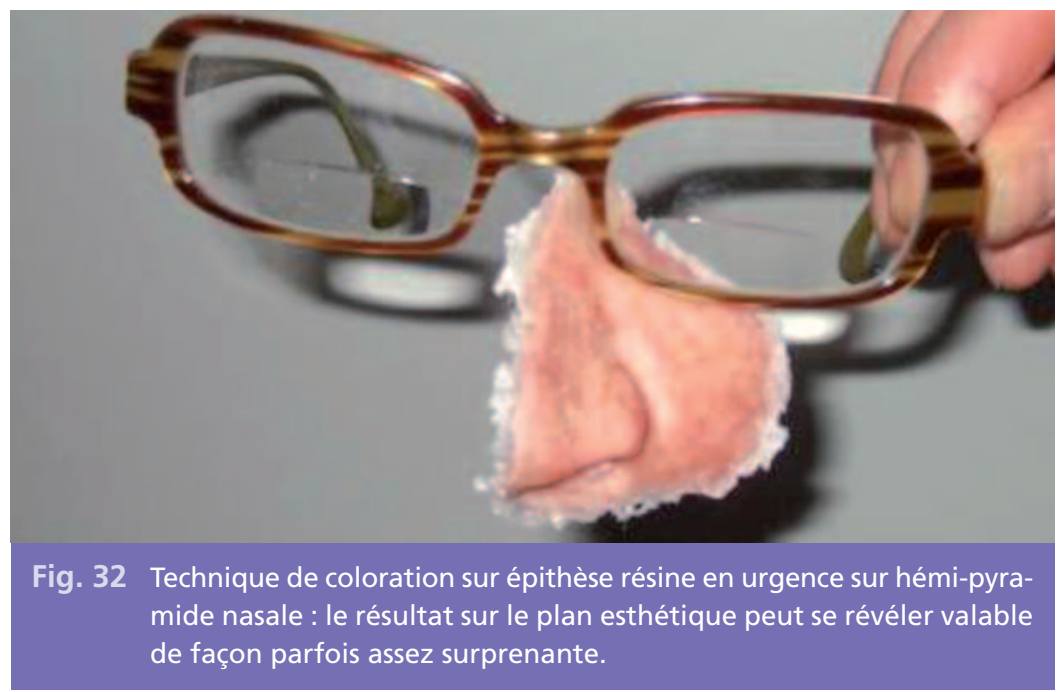


mentations, varicosités spécialement marquées chez les sujets ayant subi une radiothérapie de la sphère oro-faciale.

En ce qui concerne le système pileux, le Dr Jacques Dichamp recommande l'astuce de demander au patient de recueillir dans son rasoir électrique de la barbe qui sera ensuite saupoudrée sur la prothèse grâce à une salière. Cela évite ainsi le fastidieux travail de pointillisme obtenu en mélangeant du bleu de cobalt avec une pointe de jaune japonais citron ou de cadmium.

Notons que les varicosités peuvent être rendues avec vérité par l'utilisation d'un flockage rouge saupoudré en surface ou incorporé dans la prothèse.

Enfin les bords seront vérifiés afin de pouvoir apprécier s'ils se fondent de façon acceptable dans la teinte des tissus environnants. On terminera la prothèse comme on terminerait un tableau avec une couche de vernis final à l'aide du mélange floc marron et silicone medical adhésive A. Cette façon de procéder permet, rappelons-le, de matifier la prothèse en silicone ou en résine acrylique (fig. 33).

Il convient tout au long de ces étapes d'obtenir un mimétisme acceptable, la prothèse se devant d'être discrète sur le visage reconstitué de notre patient. Et il convient de prêter attention en particulier au léger virage de teinte lors du séchage car le monomère ne se comporte pas comme de l'huile de lin mélangée à de l'essence de térébenthine mais tend à foncer au séchage avec toute couleur déposée sur l'épithèse. II faut

\section{Conclusion}

Comme chez les peintres qui possèdent du " métier », l'ébauche joue un rôle considérable en restant visible par endroit. alors passer au papier de verre la surface pour retrouver peu ou prou la teinte de base et recommencer. En effet, travailler en synthèse soustractive impose que l'ajout de pigments nous entraîne à obtenir des teintes plus foncées qu'elles ne le devraient, erreur qu'il est très difficile de rattraper.

Ces pratiques sont valables aussi bien pour les patients de type caucasien que ceux de type mélanoderme. Mais ces derniers ont des teintes de base plus difficiles à cerner pour nos yeux car l'être humain discerne moins bien les couleurs sombres que lumineuses. En conséquence, il convient de vivement les éclairer afin d'en distinguer la teinte de base et de se méfier plus encore chez eux d'un virage foncé au séchage qui forcerait à tout reprendre depuis le début.

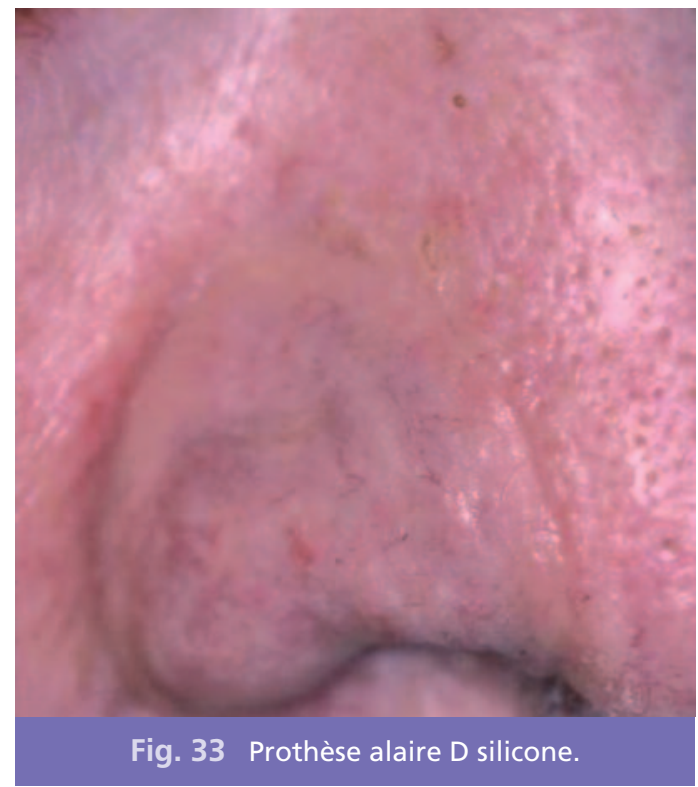

Cette ébauche correspondra à la prothèse sortie du moufle dans une teinte de base et cela, quel que soit le matériau, silicone ou résine 
acrylique, et la technique, simple ou galvanoplastie.

Par prudence, il vaut mieux choisir la teinte de base plus claire que la teinte observée sur le patient en éclairage "lumière du jour ". La technique par vitropression est un moyen utile qu'il faut savoir dépasser avec l'expérience. Plus on se rapprochera de la teinte exacte du patient, moins nombreuses seront les retouches ultérieures. Certains prothésistes sortent directement une pièce acceptable d'emblée lors de la mise en place sur le patient, se limitant alors à un simple travail de finition.

Les zones les plus claires et les zones les plus sombres s'opèrent en dernier lieu, le glaçage de couleurs sombres sur des couleurs claires produit l'illusion des rides qu'il convient quand même de sculpter. Ainsi la technique du

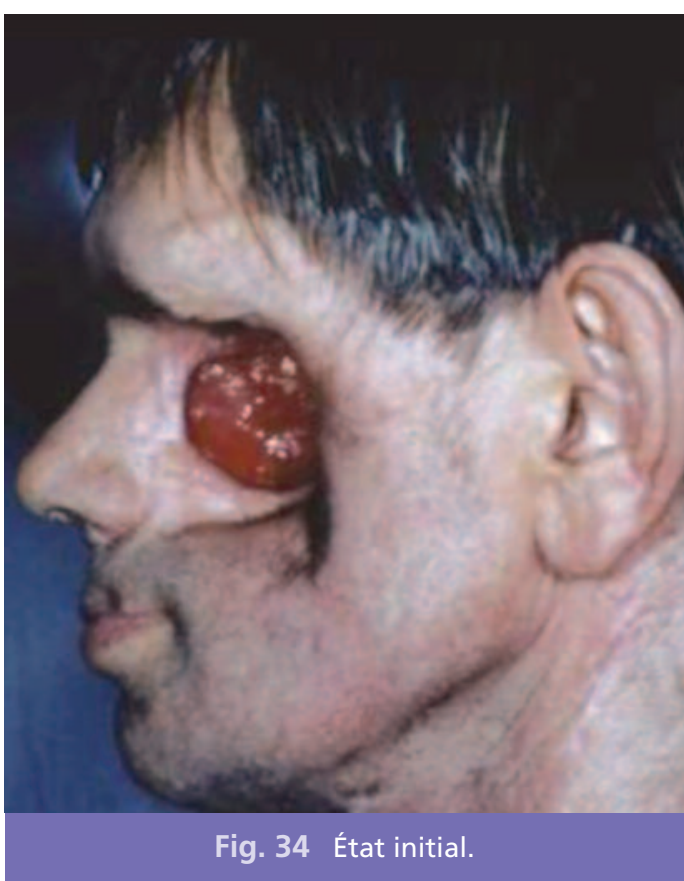

trompe-l'œil accentuera l'impression de relief de la pièce prothétique.

Les reprises doivent être limitées au maximum à cause de l'assombrissement inhérent à la technique soustractive, couleurs dites " rabattues", difficile à déceler visuellement mais qui conduit inexorablement à une destruction du mimétisme.

Lors de la séance de peinture au fauteuil, la prothèse étant en place, il conviendra d'aller toujours droit à l'essentiel et de se rappeler cet aphorisme de John Singer : "Dans l'Art, tout ce qui n'est pas indispensable est préjudiciable ». II faudra bien avoir à l'esprit de réfléchir constamment à la couleur nécessaire pour arriver à la teinte que l'on désire avoir à partir d'une teinte donnée, ce qui caractérise la plus exacte compréhension du cercle chromatique.

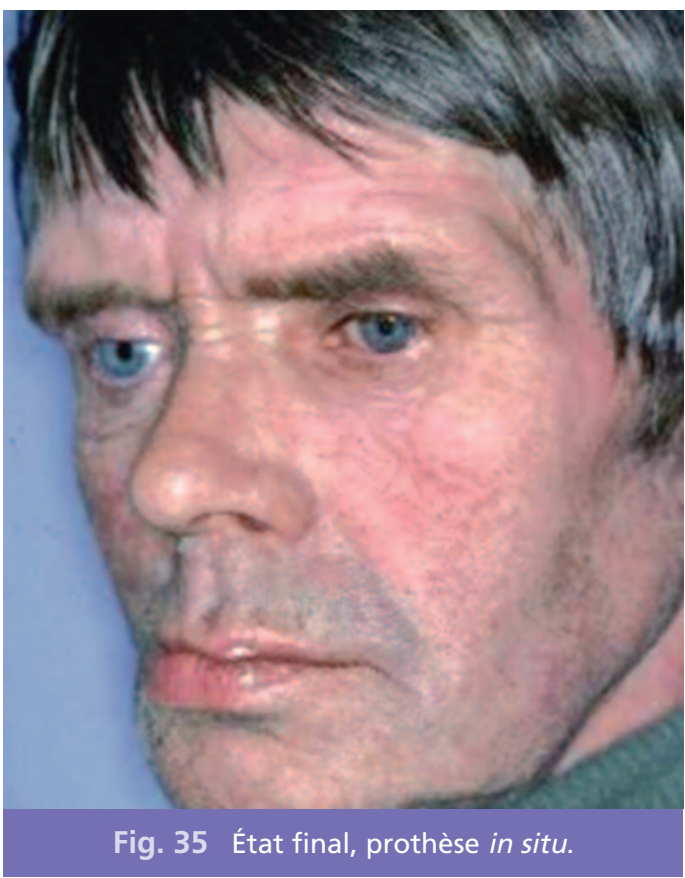

Nous remercions Gérard Poulain, prothésiste à I'UFR 056 de stomatologie et de chirurgie maxillo-faciale à I'hôpital de la Pitié-Salpêtrière, pour la réalisation clinique des travaux présentés dans cette dernière partie de notre article, lors du diplôme de Prothèse faciale appliquée. 


\section{Ouvrages à consulter}

- Benoist M.

Réhabilitation et prothèse maxillo-faciales.

Paris : Julien Prélat, 1978.

- Benoist M, Quentin Y Histoire de la prothèse maxillo-faciale. Act Odonto Stomatol 1998;202:179-93.

- Dichamp J, Guilbert F, Vaillant JM.

Prothèse plastique faciale. Encycl Med Chir (Elsevier SAS, Paris),

Stomatologie, 22-087-M-10, 1990.

- Quentin PY.

Prothèses nasales.

Revue Française

de Prothèse Maxillo-Faciale 1979 juin;III(2):72-82.

- Gary JJ, Smith CT.

Pigments and their application in maxillofacial elastomers :

a literature review.

J Prosthet Dent 1998;80(2):204-8.

- Ma T, Hicken SC, Buchanan CR, DeBoie RG.

Chairside color verification for facial prostheses.

J Prosthet Dent 1988;60(2):219-21.

- Van Volen L.

Prothèses plastiques complexes et leurs problèmes esthétiques. Revue Française de Prothèse Maxillo-Faciale 1975 déc.;IV(4):219-24.

- Galton J.

The Encyclopedia of oil painting techniques.

London: Quarto Publishing PLC, 1991.

- Moran R.

Secrets de peintres.

Technique : les glacis.

Paris : Fleurus, 1994:125.
- Yvel C.

Peindre à I'huile

comme les maîtres.

Les glacis.

Aix-en-Provence : Edisud

2003:139.

- De Langlais X.

La technique de la peinture à I'huile.

Paris : Flammarion, 1992.

- Delamarre F, Guineau B.

Les matériaux de la couleur.

Paris : Découvertes Gallimard, 1999.

- Garcia P..

Le métier de peintre.

Paris : Dessain et Tolra, 1990.

- Montchaud R.

La couleur et ses accords.

Paris : Fleurus, 1994.

- Lefranc \& Bourgeois.

Guide de la peinture à I'huile.

Le Mans : Lefranc \& Bourgeois.

\section{NOWVLLE REVUE}

\section{Droit et Médecine Bucco-Dentaire La revue de l'actualité en droit dentaire}

Directeur de la rédaction : Alain Béry

Rédacteurs en chef : Laurent Delprat et Charles Georget

Il y a plusieurs années, sous l'impulsion d'André Demichel, est apparue l'émergence du droit médical. Désormais, devant la spécialisation croissante des techniques et du droit, l'instauration d'un droit dentaire spécifique semble relever de l'évidence.

C'est dans cet esprit de reconnaissance que I'Association de Droit Dentaire, portée par l'indéfectible soutien de sa Présidente d'honneur Francine Demichel, a souhaité procéder à la création de cette nouvelle revue de "Droit et Médecine Bucco-Dentaire".

La publication, trimestrielle, permet de rendre compte rapidement de l'actualité juridique, qu'il s'agisse de nouvelles lois ou jurisprudences, réformes, études de cas, ou autres décisions touchant votre exercice quotidien.

$$
\text { Abonnez-vous en ligne! }
$$

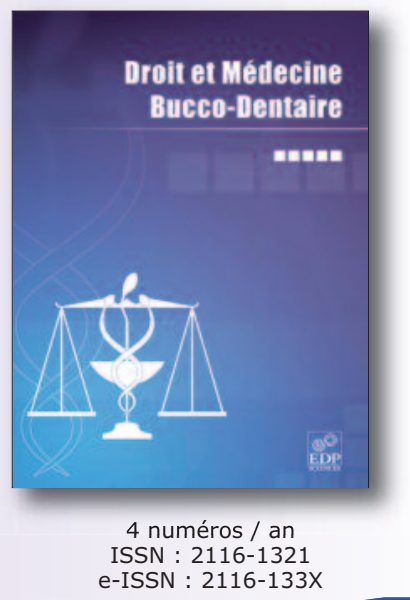

